ARTICLE

\title{
Probing resonating valence bond states in artificial quantum magnets
}

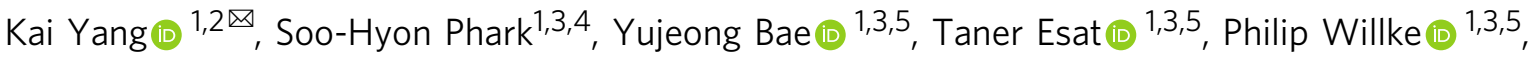 \\ Arzhang Ardavan (D) 6, Andreas J. Heinrich (10 ${ }^{3,4 凶} \&$ Christopher P. Lutz (D) ${ }^{1 \times}$
}

Designing and characterizing the many-body behaviors of quantum materials represents a prominent challenge for understanding strongly correlated physics and quantum information processing. We constructed artificial quantum magnets on a surface by using spin-1/2 atoms in a scanning tunneling microscope (STM). These coupled spins feature strong quantum fluctuations due to antiferromagnetic exchange interactions between neighboring atoms. To characterize the resulting collective magnetic states and their energy levels, we performed electron spin resonance on individual atoms within each quantum magnet. This gives atomicscale access to properties of the exotic quantum many-body states, such as a finite-size realization of a resonating valence bond state. The tunable atomic-scale magnetic field from the STM tip allows us to further characterize and engineer the quantum states. These results open a new avenue to designing and exploring quantum magnets at the atomic scale for applications in spintronics and quantum simulations.

\footnotetext{
${ }^{1}$ IBM Almaden Research Center, San Jose, CA, USA. ${ }^{2}$ Beijing National Laboratory for Condensed Matter Physics and Institute of Physics, Chinese Academy of Sciences, Beijing, China. ${ }^{3}$ Center for Quantum Nanoscience, Institute for Basic Science (IBS), Seoul, Republic of Korea. ${ }^{4}$ Department of Physics, Ewha Womans University, Seoul, Republic of Korea. ${ }^{5}$ Ewha Womans University, Seoul, Republic of Korea. ${ }^{6}$ CAESR, Clarendon Laboratory, Department of Physics,

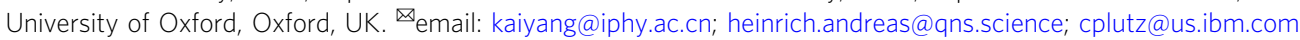


A ntiferromagnetism is classically described by a Néel state with alternating spin orientations on neighboring atoms. However, in low-dimensional and low-spin antiferromagnets, quantum spin fluctuations are enhanced and tend to suppress the classical Néel order. This gives rise to exotic ground states lacking long-range magnetic order, such as quantum spin liquids ${ }^{1}$. A class of spin liquids is characterized by the resonating valence bond (RVB) state ${ }^{2}$, in which the quantum spins continuously alter their singlet partners. RVB states have been a central topic in quantum magnetism ${ }^{3-5}$ because they are believed to describe spin-1/2 Mott insulators such as the undoped copper oxides in high- $T_{\mathrm{c}}$ superconductors ${ }^{6}$. The RVB-type spin liquid has been observed by probing the spinon excitations using neutron scattering ${ }^{7-9}$ and nuclear magnetic resonance ${ }^{10}$. The lowenergy excitations from the RVB states are fractional quasiparticles such as spinons and holons that enable spin-charge separation $^{11,12}$ and result in low-loss spin transmission through insulators ${ }^{11}$.

RVB states in finite-sized spin arrays exhibit several important spin liquid properties such as a singlet ground state lacking conventional magnetic order, strong entanglement, and a stabilizing energy benefit from coherent RVB fluctuations ${ }^{4,5}$. Experimentally, these finite-size RVB states have been created in ensembles of ultracold atoms in optical lattices ${ }^{5}$, and in entangled photons ${ }^{4}$, providing new insights into the quantum correlations.
However, RVB states with single-spin addressability have not yet been realized in a solid-state environment.

Atomic-scale magnetic structures such as spin chains and arrays have been constructed on surfaces using large-spin atoms in a scanning tunneling microscope $(\mathrm{STM})^{13-16}$. Recently, the high energy resolution of magnetic resonance was combined with STM to coherently probe the states of individual spins ${ }^{17}$ and spin pairs on a surface $18-22$. Here, we used STM to perform electron spin resonance (ESR) of designed quantum magnets, in which we use spin-1/2 atoms to obtain strong quantum fluctuations, and we measured their many-body states with atomic resolution (Fig. 1a). This allows for the exploration of magnetic behaviors such as RVB states, which exhibit different degrees of quantum fluctuation and different excitation properties depending on their geometry. These quantum magnets provide a versatile quantum matter toolkit with atom-selective spin resonance spectroscopy that allows highly entangled states to be generated and probed in detail.

\section{Results}

Spin Hamiltonian of quantum magnets. Each quantum magnet was made by positioning $\mathrm{Ti}$ atomic spins on an insulating $\mathrm{MgO}$ film. Each Ti atom is hydrogenated, making it part of a $\mathrm{TiH}$ molecule ${ }^{20,23}$ and for simplicity, we refer to it simply as a $\mathrm{Ti}$

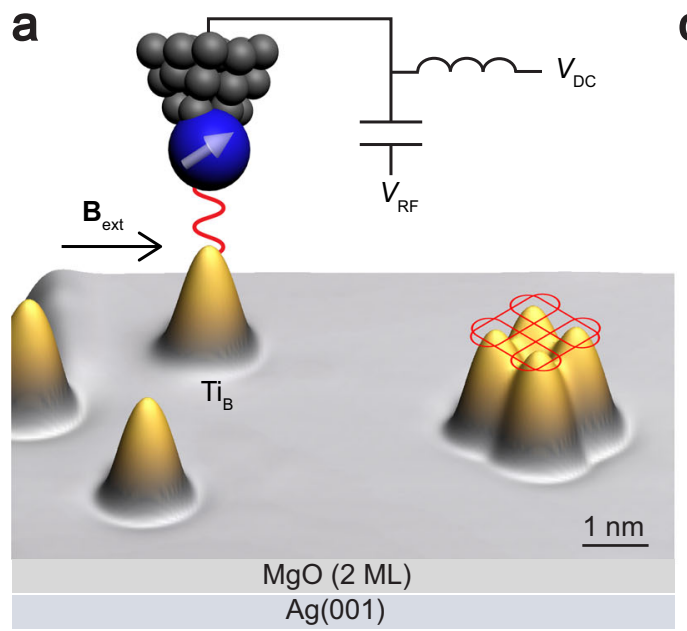

b

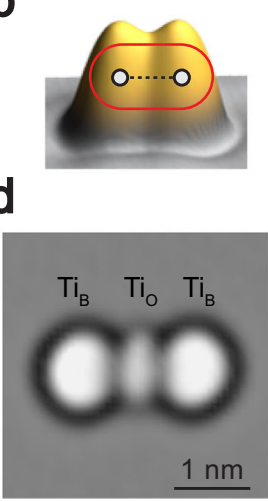

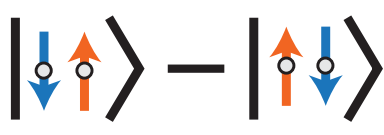

valence bond

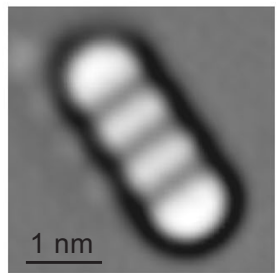

C

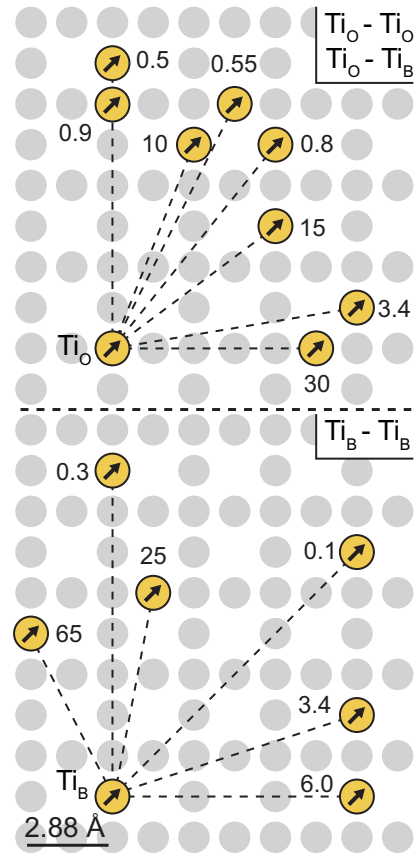

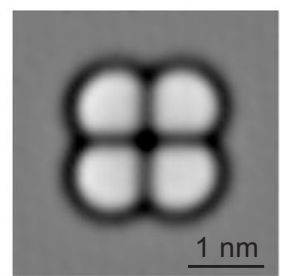

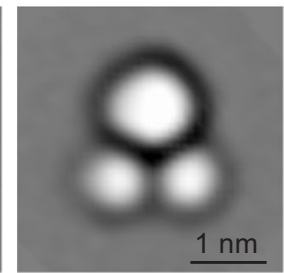

Fig. 1 Toolbox of spin-1/2 atoms on a surface. a Experimental setup showing an STM with ESR capability, and an STM image (10 $\times 7.8 \mathrm{~nm})$ of Ti atoms on $\mathrm{MgO} / \mathrm{Ag}(001)\left(V_{D C}=50 \mathrm{mV}, I_{D C}=30 \mathrm{pA}\right)$. A spin-polarized tip is used to drive and sense ESR. Rounded rectangles represent valence bonds between Ti atoms positioned on the surface. $\mathbf{b}$ Valence bond (spin-singlet state), formed by two antiferromagnetically coupled Ti atoms. Blue and orange arrows represent spin-down and spin-up states, respectively. c Pairwise magnetic interactions (in units of $\mathrm{GHz}$ ) for a range of different interatomic distances (Supplementary Table 1). Upper panel: $\mathrm{Ti}_{\mathrm{O}}-\mathrm{Ti}_{\mathrm{O}}$ and $\mathrm{Ti}_{\mathrm{O}}-\mathrm{Ti}_{\mathrm{B}}$ dimers. Lower panel: $\mathrm{Ti}_{\mathrm{B}}-\mathrm{Ti}_{\mathrm{B}}$ dimers. $\mathbf{d}$ Laplace-filtered $\mathrm{STM}$ images $(3.5 \times 3.5 \mathrm{~nm}$ ) of different quantum magnets. 
atom. Each $\mathrm{Ti}$ atom was adsorbed either on top of an oxygen atom $\left(\mathrm{Ti}_{\mathrm{O}}\right)$ or at a bridge site $\left(\mathrm{Ti}_{\mathrm{B}}\right)$ between two oxygen atoms ${ }^{20,21}$. At both sites it has spin $S=1 / 2^{20,21}$, giving it negligible magnetocrystalline anisotropy ${ }^{24}$ that could suppress spin fluctuations. Due to the antiferromagnetic exchange interaction at close distance ${ }^{20,21}$, a pair of Ti spins forms a singlet ground state $\circ-\circ=\downarrow \uparrow-\uparrow \downarrow$ (normalization factors omitted throughout) (Fig. 1b), known as a valence bond $d^{5,6}$. Here $\uparrow$ and $\downarrow$ represent spin-up and spin-down states, respectively. In structures containing more than two spins, quantum fluctuations between many such valence bonds can give rise to the RVB state, in which the spins continuously alter their singlet partners and rearrange the pairings.

To design a quantum magnet exhibiting RVB states, we first characterized the pairwise magnetic interaction $J$ for different $\mathrm{Ti}$ pairs by measuring the splitting of ESR peaks as a function of distance ${ }^{20}$, as shown in Fig. 1c. We used the Ti spins coupled dominantly by antiferromagnetic exchange $\left(J_{i j}>0\right)$ to build quantum magnets, including odd- and even-length spin chains, spin triangles, and spin plaquettes (Fig. 1d and Supplementary Fig. 1). The quantum states of these quantum magnets under external applied magnetic field $\mathbf{B}_{\text {ext }}$ (in-plane) are described by the Hamiltonian ${ }^{20,25}$ :

$$
H=\sum_{i, j} J_{i j} \mathbf{S}_{i} \cdot \mathbf{S}_{j}+\sum_{i} g \mu_{\mathrm{B}} \mathbf{B}_{\mathrm{ext}} \cdot \mathbf{S}_{i}+g \mu_{\mathrm{B}} \mathbf{B}_{\mathrm{tip}} \cdot \mathbf{S}_{n}
$$

where $\mathbf{S}_{i}$ is the spin operator for site $i$. The g-factor $g \approx 1.8$ is obtained by ESR of isolated Ti atoms ${ }^{20,25}$, and $\mu_{\mathrm{B}}$ is the Bohr magneton. The antiferromagnetic interactions compete with the Zeeman term, which tends to align the spins along $\mathbf{B}_{\text {ext }}$. Here, we used an isotropic spin-1/2 model as an approximation which agrees well with most of the experimental data and thus captures the main physics. Consideration of g-factor anisotropy ${ }^{23}$ could further improve the accuracy of the spin Hamiltonian. Note that $\mathbf{B}_{\text {ext }}$ is the only source of anisotropy in the Hamiltonian, so changing its direction (here it is applied in-plane) should not affect the form of the eigenstates or energies. The atomic-scale tip magnetic field $\mathbf{B}_{\text {tip }}$ (Supplementary Fig. 2) is used both to drive ESR transitions and to tune the spin states of the quantum magnets by exerting an exchange bias only on the spin $\mathbf{S}_{n}$ under the tip ${ }^{25}$. Here, the ESR transitions between two coupled-spin states $|i\rangle$ and $|j\rangle$ are allowed if there is a nonzero matrix element $\left\langle i\left|\Delta \mathbf{B}_{\text {tip }} \cdot \mathbf{S}_{n}\right| j\right\rangle$, where $\Delta \mathbf{B}_{\text {tip }}$ gives the field gradient ${ }^{20}$. We are thus able to drive ESR transitions between different spin multiplets, which are forbidden in traditional spin resonance ${ }^{26}$, offering direct access to the energy differences between multiplets.

RVB states in an odd number chain. We first built a three-spin chain by alternating $\mathrm{Ti}_{\mathrm{O}}$ and $\mathrm{Ti}_{\mathrm{B}}$ atoms to obtain the nearestneighbor coupling of $J \approx 30 \mathrm{GHz}$ (Fig. 2). This coupling strength results in a low-spin ground state, while allowing transitions between different multiplets to be visible in our ESR range of $\sim 10-30 \mathrm{GHz}$. Each spin multiplet with a total spin $S_{\mathrm{T}}$ fans out into its $2 S_{\mathrm{T}}+1$ components, each having a different quantum number $M$, when $\mathbf{B}_{\text {ext }}$ is increased (Fig. 2a and Supplementary Fig. 3). The three-spin chain has a ground state $|1\rangle=2(\downarrow \uparrow \downarrow)-(\downarrow \downarrow \uparrow+\uparrow \downarrow \downarrow)=\circ-\downarrow \downarrow-\downarrow \circ-\circ$. This coherent superposition of valence bond states is a resonance between $\circ-\downarrow$ and $\downarrow \circ-$, giving an energy of $-J$, which is lower than the energy expectation for states having only one valence bond ( $-\llcorner\downarrow$ or $\downarrow \circ-\circ)$ or for a Néel state $(\downarrow \uparrow \downarrow)$. The case of a single valence-bond state would arise in the limiting case where $J_{12} \gg J_{23}$. The extreme case is the third spin being isolated from the other two spins, and evolution of the ESR spectra measured on the middle spin would be the same as those measured on a
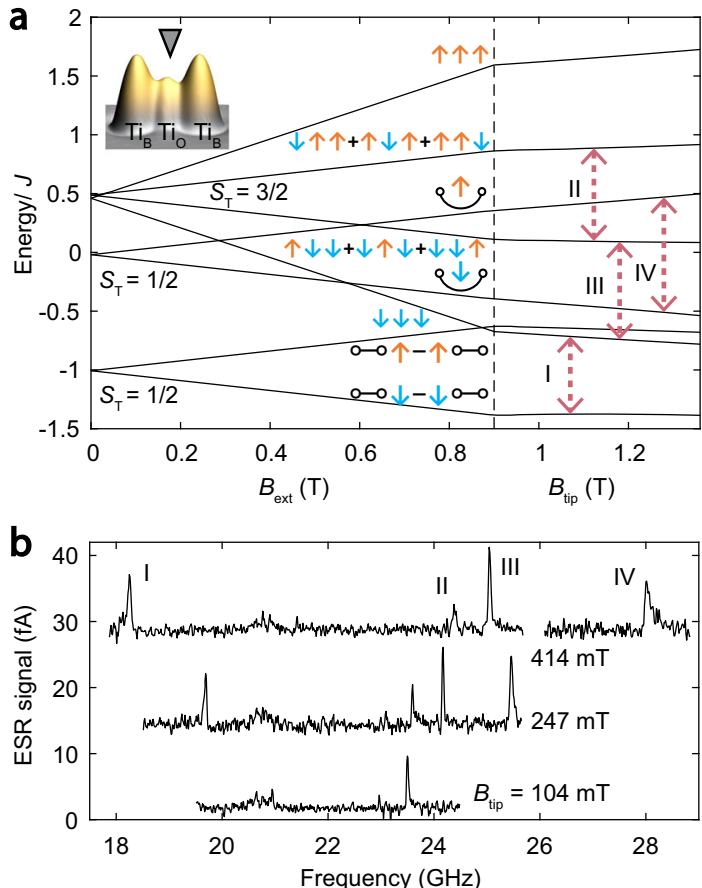
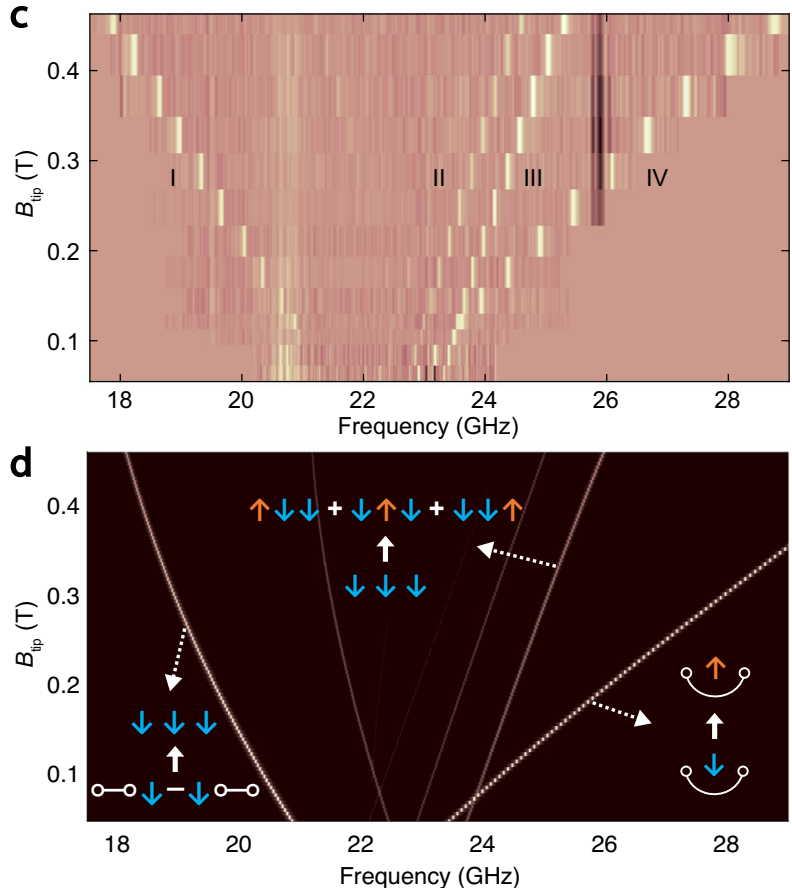

Fig. 2 Magnetic states of a three-spin chain. a Energy level diagram as a function of $B_{\text {ext }}$ (up to $0.9 \mathrm{~T}$ ), and $B_{\text {tip }}$ with $J \approx 30 \mathrm{GHz}$, calculated using

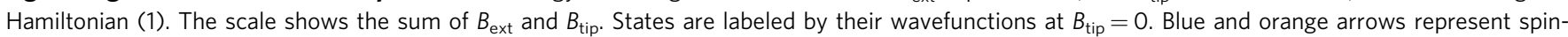
down and spin-up states, respectively (same below). Dashed arrows indicate detected ESR transitions. b, $\mathbf{c}$ ESR spectra measured on the middle (TiO) spin at different $B_{\text {tip }}$ as labeled, shown as a graph (b) and lightness scale $(\mathbf{c})$. $\left(V_{\mathrm{DC}}=60 \mathrm{mV}, I_{\mathrm{DC}}=5-40 \mathrm{pA}, V_{\mathrm{RF}}=7-22 \mathrm{mV}, B_{\mathrm{ext}}=0.9 \mathrm{~T}\right)$. Signals near 20.6 and $25.9 \mathrm{GHz}$ are experimental artifacts due to the frequency-dependent RF transmission. d, Simulated ESR spectra. Weak anisotropy 48 ( $-0.02 \mathrm{~J}$ ) is added to the exchange Hamiltonian to reproduce the observed slightly broken degeneracy, yielding distinct peaks II and III (Supplementary Note 5). 
spin dimer ${ }^{21}$. In contrast, Néel states should appear only for spins having large enough magnetic anisotropy ${ }^{27}$ or in arrays having sufficient disorder to mix the RVB state with higher-lying states. This 3-spin chain represents the simplest example of an RVB state, illustrating how a coherent superposition of valence bond states gives an energy benefit.

To probe the energy spectrum and eigenstates of this threespin chain, we performed ESR on the middle spin and studied the evolution of the ESR spectra as a function of $B_{\text {tip }}$ (Fig. 2b, c). Since different ESR transitions respond differently with increasing $B_{\text {tip }}$, this allows us to easily identify the corresponding initial and final states, in accordance with the quantitative simulations (Fig. 2d).

For example, the lowest-frequency ESR peak (I) reveals the transition from the ground state $|1\rangle=\circ \multimap \downarrow-\downarrow \circ \bigcirc\left(S_{\mathrm{T}}=1 / 2\right.$, $M=-1 / 2)$, to the ferromagnetic state $|2\rangle=\downarrow \downarrow \downarrow\left(S_{\mathrm{T}}=3 / 2, M=\right.$ $-3 / 2$ ). The average spin polarization $\left\langle S_{2}^{z}\right\rangle$ (where $z$ is the direction of $\mathbf{B}_{\text {ext }}$ ) of the middle spin is zero in the state $|1\rangle$, while in state $|2\rangle$ it is fully polarized. This difference in local spin polarization is directly visualized as the frequency shift of peak I with increasing $B_{\text {tip }}$, which favors the spin polarization of the middle spin in state $|2\rangle$. For the higher-energy states $|4\rangle=q \downarrow g$ and $|6\rangle=£$, the two end spins form a spin-singlet. These two states are also eigenstates of the spin Hamiltonian having zero energies at zero magnetic field since the direct coupling between the two end spins is negligible. The polarization of the center spin is opposite for these two states, so the corresponding ESR transition (IV) shifts to higher frequency rapidly as we increase $B_{\text {tip }}$.
The observation of the ESR transitions I and III allows us to determine the energy of the RVB ground state with respect to the ferromagnetic state, which is a conventional measure of RVB energy ${ }^{28}$ that gives the quantitative energy benefit for forming this coherent superposition of singlets. Here, it is given by $-\left(f_{\mathrm{I}}+f_{\text {III }}\right) / 3=(-0.497 \pm 0.010) J$ per spin at zero magnetic field (Supplementary Fig. 5), in agreement with the calculated value of $-0.5 \mathrm{~J}$.

RVB states in an even number chain. Quantum magnets having an even number of spins ${ }^{14}$ can exhibit a non-magnetic RVB ground state because all the spins can simultaneously pair into valence bonds. Resonance among different ways to pair the spins results in intriguing spin entanglement between pairs that are not directly coupled ${ }^{4,29,30}$. To explore these properties, we built a four-spin chain with a strong pairwise coupling of $\sim 65$ $\mathrm{GHz}$ (Fig. 3a), which exceeds the Zeeman energy of individual spins at $0.9 \mathrm{~T}$ so that the RVB state, which is non-magnetic $\left(S_{\mathrm{T}}\right.$ $=0$ ), becomes the ground state (Fig. $3 \mathrm{~b}$ and Supplementary Fig. 8). The RVB state is the superposition of two pairing configurations: $\quad\left(\downarrow_{1} \uparrow_{2}-\uparrow_{1} \downarrow_{2}\right)\left(\downarrow_{3} \uparrow_{4}-\uparrow_{3} \downarrow_{4}\right) \quad$ and $\left(\downarrow_{1} \uparrow_{4}-\uparrow_{1} \downarrow_{4}\right)\left(\downarrow_{2} \uparrow_{3}-\uparrow_{2} \downarrow_{3}\right)$ (Fig. 3a, bottom). Even though there is negligible direct pairwise interaction between the end spins $\mathbf{S}_{1}$ and $\mathbf{S}_{4}$, the singlet bonding configuration for these spins still appears in the ground state. Thus, the RVB state can be envisaged as a fluctuating pattern of valence bonds having different lengths ${ }^{2}$. We measured the ESR spectra on different sites as a function of $B_{\text {tip }}$ (Fig. 3c). This allowed us to extract
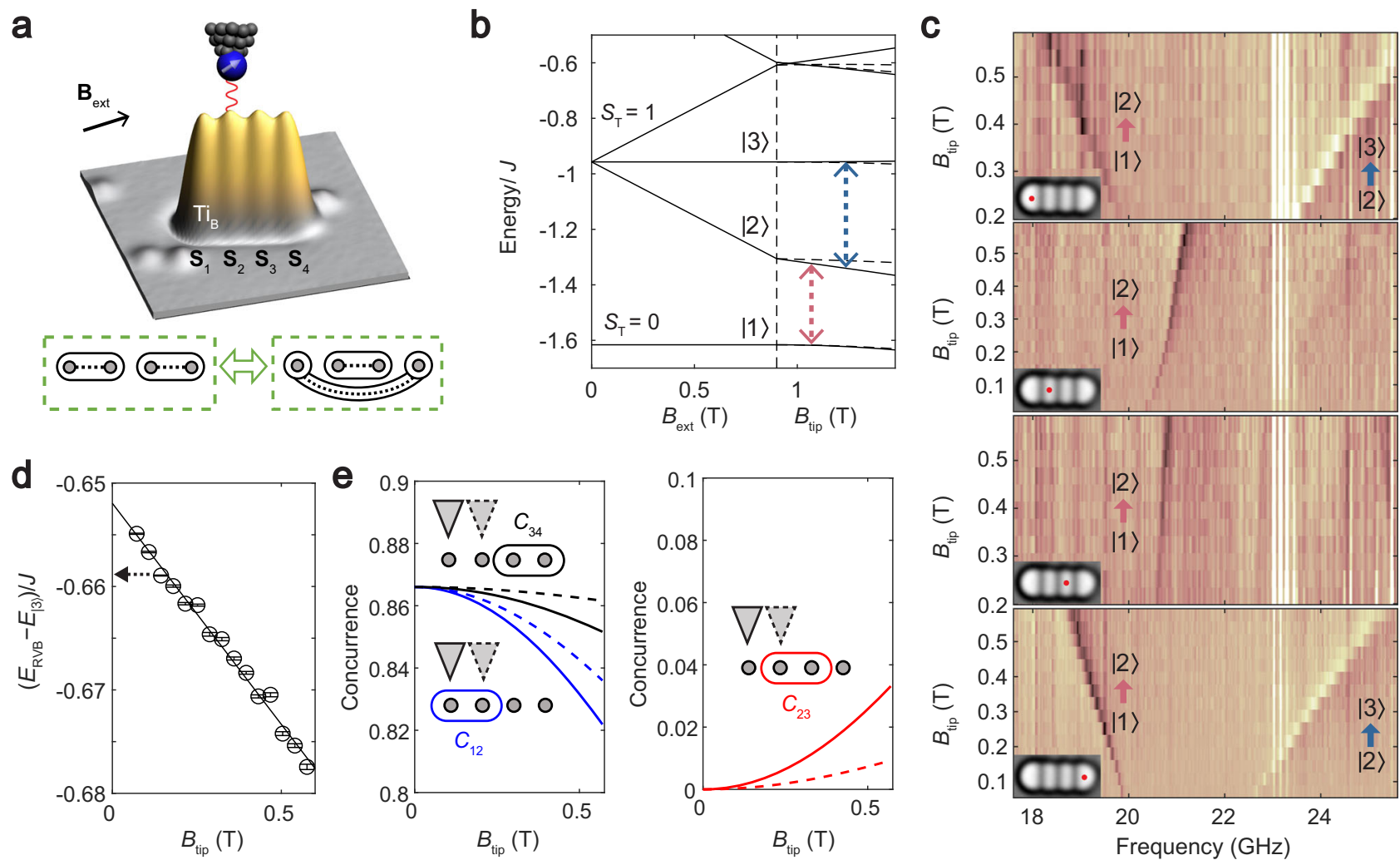

Fig. 3 Spin pairings in an even-length chain. a Experimental setup showing a four-spin chain of $\mathrm{Ti}_{\mathrm{B}}$ atoms $(J \approx 65 \mathrm{GHz}$ ). The double arrow indicates the superposition of two spin-pairing configurations of the ground state $|1\rangle$. $\mathbf{b}$ Energy level diagram as a function of $B_{\text {ext }}$ and $B_{\text {tip }}$ applied to $\mathbf{S}_{1}$ (solid lines) or $\mathbf{S}_{2}$ (dashed lines). Dashed arrows indicate detected ESR transitions. $\mathbf{c}$ ESR spectra measured on each of the $4 \mathrm{spins}$ as a function of $B_{\text {tip }}\left(V_{\mathrm{DC}}=60 \mathrm{mV}, I_{\mathrm{DC}}=\right.$ 10-160 pA, $\left.V_{R F}=24-38 \mathrm{mV}, B_{\text {ext }}=0.9 \mathrm{~T}\right)$. Vertical stripes are due to the frequency-dependent RF transmission. $\mathbf{d}$ Energy difference between states $|1\rangle$ and $|3\rangle$ at different $B_{\text {tip }}$, with a linear fit (solid line). Dashed arrow indicates the theoretical value at $B_{\text {tip }}=0$. Error bars are from the fitting uncertainties of the ESR frequencies with a $95 \%$ confidence. e Ground-state concurrence $C_{i j}$ between the three different pairs of neighboring spins, as a function of $B_{\text {tip. }}$ Solid (dotted) lines show concurrence when the $B_{\text {tip }}$ is applied to $\mathbf{S}_{1}\left(\mathbf{S}_{2}\right)$ as illustrated. 
information about the spin wavefunction, including spin polarization on different sites and spin pairings. Conventional tunneling spectra $(\mathrm{d} I / \mathrm{d} V)$ are thermally limited so they resolve only a single broadened excitation for this chain (Supplementary Fig. 6).

The ESR transition from the RVB ground state $|1\rangle$ to the first excited state $|2\rangle\left(S_{\mathrm{T}}=1, M=-1\right)$ evolves differently depending on where $B_{\text {tip }}$ is applied. Since all spins have zero polarization in state $|1\rangle$, this evolution gives us information about the spin distributions of the state $|2\rangle$ with atomic resolution. Examination of the quantitative spin states shows that for state $|2\rangle$ the $z$-axis spin polarization of $S_{1}(\sim 85 \%)$ is larger than $S_{2}(\sim 15 \%)$ (Supplementary Fig. 7). Accordingly, the frequency of the ESR transition from the state $|1\rangle$ to $|2\rangle$ shifts faster when $B_{\text {tip }}$ is applied to $\mathbf{S}_{1}$ than the case with $B_{\text {tip }}$ applied on $\mathbf{S}_{2}$ (Fig. $3 \mathrm{c}$ ). At higher frequency, the transition from $|2\rangle$ to $|3\rangle$ is visible when measuring the spin at either end of the chain.

This atomic-scale ESR offers a direct measurement of the energy differences between spin multiplets, due to its distinct selection rule based on atomically local excitation, while these relative energies are not accessible in conventional bulk ESR ${ }^{26}$. For example, the sum of the frequencies of the two detected ESR transitions gives the energy difference between the two lowest spin multiplets (Fig. 3d), which is $(-0.652 \pm 0.010) J$ at zero field, in agreement with the calculated value of $-0.659 \mathrm{~J}$.

The atomic-scale tip field also influences the pairwise entanglement in the RVB ground state through its competition with the exchange coupling and the external field. To qualitatively understand this competition, we plot the concurrence $C_{i j}$, a measure of entanglement ${ }^{4,29,30}$, between two spin sites $i$ and $j$ computed for the ground state (Fig. $3 e$ and Supplementary Note 4). The concurrences varied differently depending on whether $B_{\text {tip }}$ was applied on $\mathbf{S}_{1}$ or $\mathbf{S}_{2}$. This sensitivity directly reflects the spin-pairing information. The tip field only has to compete with $J \mathbf{S}_{1} \cdot \mathbf{S}_{2}$ to polarize spin 1 , while it has to compete with both $J \mathbf{S}_{1} \cdot \mathbf{S}_{2}$ and $J \mathbf{S}_{2} \cdot \mathbf{S}_{3}$ when $B_{\text {tip }}$ is applied on spin 2 . Note that the decrease of $C_{12}$ is accompanied by the increase of $C_{23}$, showing the "monogamy" of entanglement ${ }^{4,29}$. a
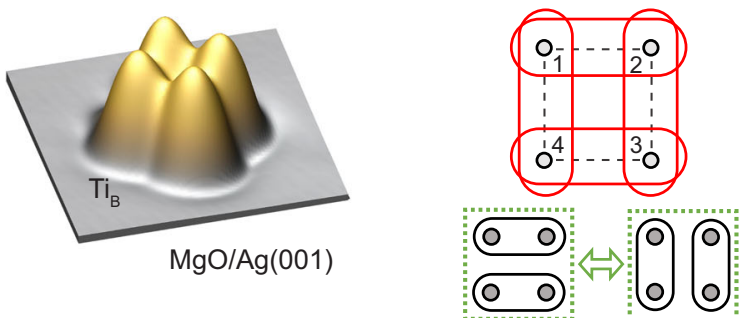

C

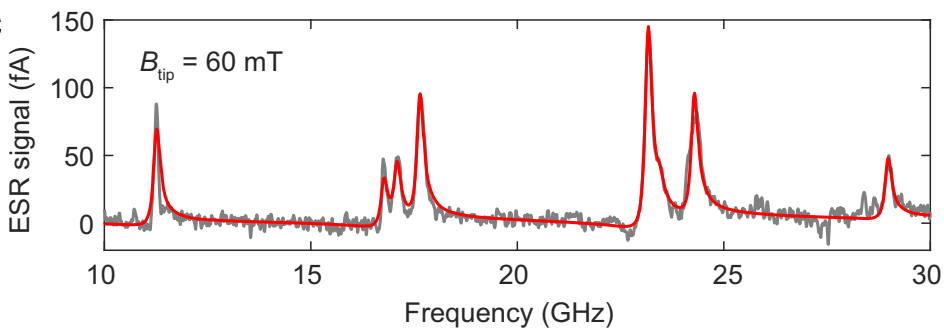

d

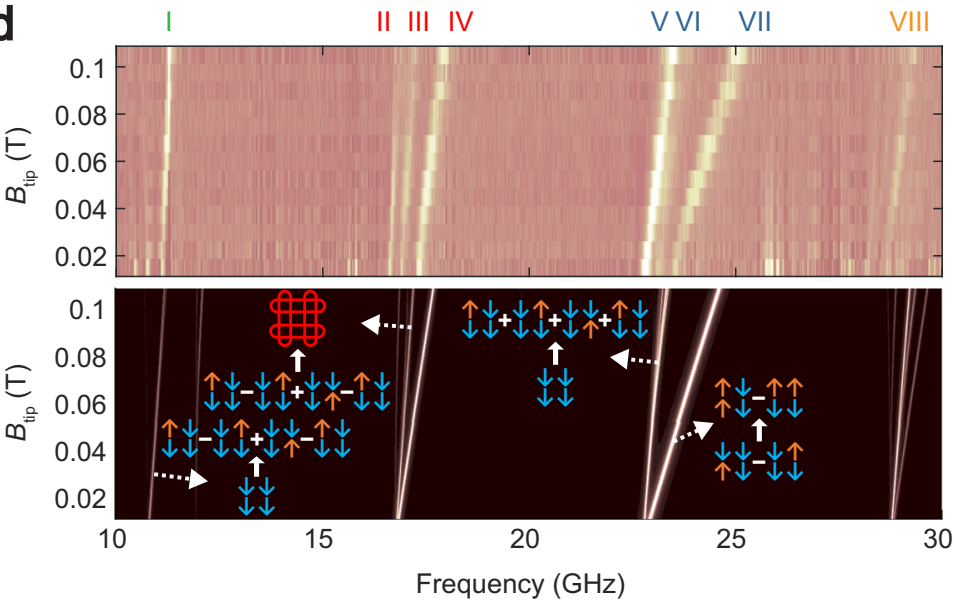

b

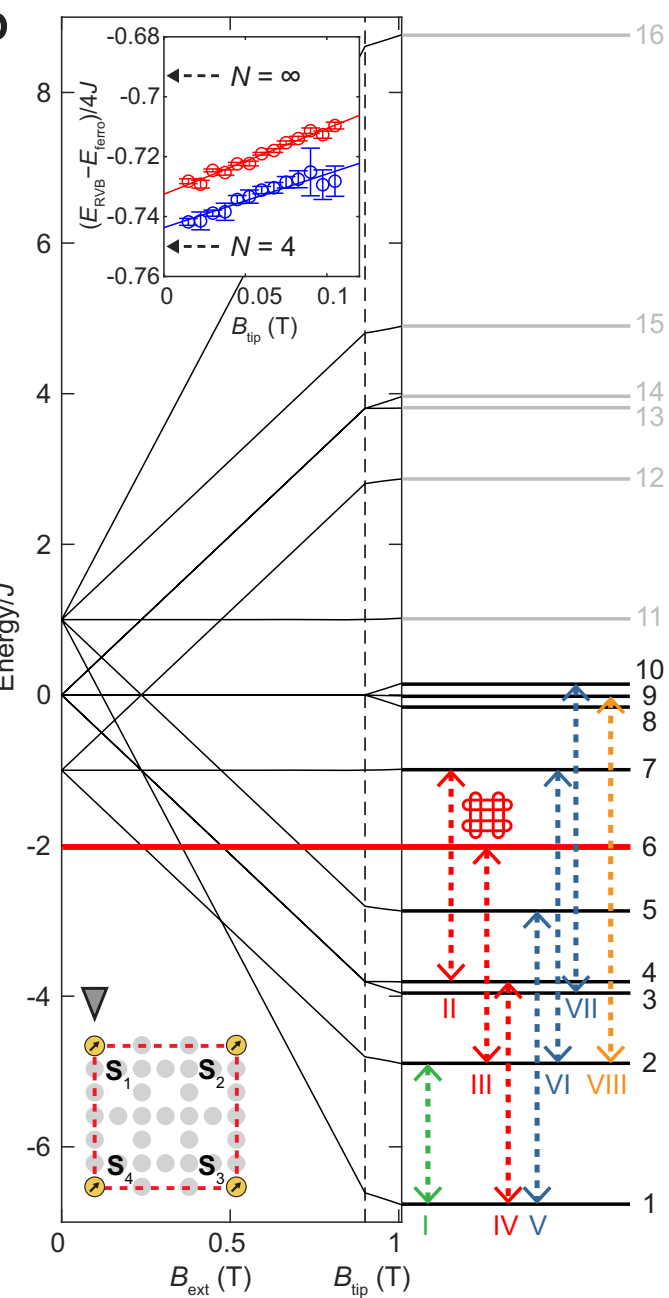

Fig. 4 RVB state in a spin plaquette. a STM image of a spin plaquette of $T_{B}$ atoms $(J \approx 6 \mathrm{GHz})$. Right: Schematic of the RVB state (red) consisting of the superposition of two spin pairings (green). $\mathbf{b}$ Energy level diagram as a function of $B_{\text {ext }}$ and $B_{\text {tip. }}$. Dashed arrows indicate detected ESR transitions. Red line is the RVB state energy. Upper inset: energy per spin of the RVB state relative to the ferromagnetic state $\downarrow_{1} \downarrow_{2} \downarrow_{3} \downarrow_{4}$ at $B_{\text {ext }}=0$, determined in two ways, by measuring $\left(f_{\mathrm{I}}+f_{\mathrm{III}}-2 g \mu_{\mathrm{B}} B_{\text {ext }}\right) / 4 J$ (red circles) or $\left(f_{\| I}+f_{\mid I I}+f_{\mathrm{IV}}-f_{\mathrm{VI}}-2 g \mu_{\mathrm{B}} B_{\text {ext }}\right) / 4 J$ (blue circles) and extrapolating to $B_{\text {tip }}=0$. Solid lines are linear fits. Dashed arrows indicate the theoretical values for $N=4$ spins and $N=\infty^{28}$ at $B_{\text {tip }}=0$. Error bars are from the fitting uncertainties of the ESR frequencies with a $95 \%$ confidence. $\mathbf{c}$ ESR spectra taken on $\mathbf{S}_{1}\left(V_{D C}=50 \mathrm{mV}, I_{D C}=200 \mathrm{pA}, V_{R F}=8 \mathrm{mV}, B_{\text {ext }}=0.9 \mathrm{~T}\right)$. Red curve is a fit to a sum of asymmetric Lorentzian peaks. d ESR spectra as a function of $B_{\text {tip }}$ of the spin plaquette $\left(V_{D C}=50 \mathrm{mV}, I_{D C}=50-350 \mathrm{pA}, V_{\mathrm{RF}}=3-13 \mathrm{mV}, B_{\mathrm{ext}}=0.9 \mathrm{~T}\right)$. Lower panel: Simulated ESR spectra. Blue and orange arrows represent spin-down and spin-up states, respectively. 
RVB states in a spin plaquette. While the four-spin chain shows an unequal superposition of the valence bond basis states, we can engineer an equal superposition by building closed-chain structures, with additional translational symmetry. A closed chain of four spins is obtained by assembling a 2-by-2 plaquette $^{31}$ (Fig. 4a), the smallest configuration for simulating a quantum spin liquid on a two-dimensional square lattice ${ }^{3-5}$. In such a geometry, there are two valence-bond basis states (Supplementary Figs. 10,11): $\left|\Phi_{=}\right\rangle=\left(\downarrow_{1} \uparrow_{2}-\uparrow_{1} \downarrow_{2}\right)\left(\downarrow_{4} \uparrow_{3}-\uparrow_{4} \downarrow_{3}\right)$ and $\left|\Phi_{\|}\right\rangle=\left(\downarrow_{1} \uparrow_{4}-\uparrow_{1} \downarrow_{4}\right)\left(\downarrow_{2} \uparrow_{3}-\uparrow_{2} \downarrow_{3}\right)$. The RVB state has $s-$ wave symmetry and consists of the coherent superposition $\left|\Phi_{=}\right\rangle+\left|\Phi_{\|}\right\rangle$, resulting in the entanglement of each spin with its two neighbors (Fig. 4a, right). The $d$-wave superposition $\left|\Phi_{\times}\right\rangle=\left|\Phi_{=}\right\rangle-\left|\Phi_{\|}\right\rangle$, in contrast, is not an RVB state and lies at higher energy (Supplementary Fig. 10).

We constructed a plaquette with an interatomic distance $d=$ $8.7 \AA ̊$ (Fig. 4a), yielding a nearest-neighbor coupling $J \approx 6 \mathrm{GHz}$, and negligible second-nearest-neighbor coupling $(\sim 0.1 \mathrm{GHz})$. At $B_{\text {ext }}=0.9 \mathrm{~T}$, the Zeeman energy dominates, and therefore the ferromagnetic state $\downarrow_{1} \downarrow_{2} \downarrow_{3} \downarrow_{4}$ is the ground state (Fig. 4b). Many transitions are accessible to ESR in such a plaquette because of the thermal occupation of several lower-lying states (Fig. 4c, d). These transitions are in excellent agreement to the energies, amplitudes, and the dependence on $B_{\text {tip }}$ given by diagonalization of the model Hamiltonian (quantum states detailed in Supplementary Fig. 11).

We obtain direct access to the RVB state by driving an ESR transition (peak III in Fig. 4) into the RVB state. We can thus measure the relative energy between the RVB state and the ferromagnetic state $\left(\downarrow_{1} \downarrow_{2} \downarrow_{3} \downarrow_{4}\right)$, giving $(-0.738 \pm 0.030) J$ per spin (Fig. 4b, upper inset). This value agrees with the expected value of $-0.75 \mathrm{~J}$ for an ideal spin- $1 / 2$ plaquette, and is much lower than the $-0.5 \mathrm{~J}$ of a Néel state $\left(\downarrow_{1} \uparrow_{2} \downarrow_{3} \uparrow_{4}\right)$, thus confirming the RVB nature of this spin plaquette. Theoretical studies show that the energy per spin decreases in magnitude with an increasing number of spins, and reaches $-0.693 \mathrm{~J}$ in the thermodynamic limit of an infinite chain ${ }^{28}$, which is remarkably similar to the four-spin value. We further brought four Ti atoms closer to form a smaller plaquette $(d=7.4 \AA)$, which increased the coupling $J$ to $25 \mathrm{GHz}$, making the RVB state lowest in energy even in the presence of the magnetic field (Supplementary Note 8).

\section{Discussion}

The formation of the RVB state studied here is due to the competition between different configurations of spin-singlet pairings. This competition exists even though the Heisenberg antiferromagnet on a square lattice does not have classical magnetic frustration, which requires odd-length cycles ${ }^{2}$. We also generated such classical frustration, coexisting with RVB behavior, by assembling Ti atoms into a spin triangle (Supplementary Note 9), and observe that the frustration brings the two low-spin doublets closer in energy than in the 3-spin chain, leading towards the high-degeneracy ground state characteristic of frustrated systems. Geometrically frustrated lattices with competing interactions are highly challenging for analytical and numerical studies. Employing the atomic-scale ESR technique developed here for probing coupled-spin states, and using a different decoupling layer such as hexagonal boron nitride, one could measure the emergent non-trivial phases of the spin-1/2 triangular lattice.

Custom-designed quantum magnets assembled on a surface combined with single-atom ESR provide a flexible platform to explore the quantum states of finite-size spin systems. This technique can introduce precisely characterized disorder by placing point defects, vacancies, and adjusted couplings by repositioning the atoms. These artificial nanomagnets could aid in the design and complement the use of chemically synthesized molecular nanomagnets ${ }^{32-34}$, which have emerged as promising vehicles for spintronics ${ }^{35}$, quantum computing $34,36,37$, and quantum simulations ${ }^{38}$. The precisely engineered finite-size quantum many-body systems demonstrated here may serve as versatile analog quantum simulators $31,34,38,39$ because they can be assembled, modified, and probed in situ with single-spin selectivity. The spin plaquette constructed here is the fundamental building block of the square lattice spin liquids ${ }^{40}$. A unique opportunity provided by the STM is to explore the real-space response of the spin liquid to point defects such as individual pinned magnetic moments, which can better reveal the character of the quantum spin liquids ${ }^{41,42}$. In addition, studies of larger spin arrays or using different atom species with larger single-ion anisotropy should allow exploration of the quantum-classical-transition, and of competition between quantum fluctuations and Néel order ${ }^{8,43}$. Another natural extension of the current work is to use atomic spins on $\mathrm{MgO}$ to realize simulations of the magnetic phases of the Mott insulating states in copper oxide high- $T_{\mathrm{c}}$ superconductors, and this could also provide a realization of the deconfined quantum critical point by tuning the quantum magnets ${ }^{44}$.

In addition to exhibiting stationary magnetic orderings, quantum spin arrays can also carry spinon spin current based on quantum fluctuations ${ }^{11}$. The combination of pump-probe electronic pulses ${ }^{45}$ with pulsed $\mathrm{ESR}^{22}$ could allow further exploration of the quantum dynamics of quasiparticles in artificial spin structures with atomic resolution, including, for example, the dynamical evolution of the spin transmission in spin arrays on surfaces, or the operation of nanoscale devices based on spin currents in insulators ${ }^{11,46}$. Studying the time evolution and confinement of these elementary excitations in $1 \mathrm{D}$ and $2 \mathrm{D}$ could help to reveal how information propagates in many-body systems, complementing numerical simulations and analytical studies ${ }^{47}$.

\section{Methods}

Sample preparation. Measurements were performed in a home-built ultrahighvacuum $\left(<10^{-9}\right.$ Torr) STM operating at $1.2 \mathrm{~K}$. MgO is two monolayers (ML) thick (referred to as bilayer $\mathrm{MgO}$ ) and was grown on an atomically clean $\mathrm{Ag}(001)$ single crystal by thermally evaporating $\mathrm{Mg}$ in an $\sim 10^{-6}$ Torr $\mathrm{O}_{2}$ environment ${ }^{17}$. Ti and $\mathrm{Fe}$ atoms were deposited in situ from pure metal rods by e-beam evaporation onto the sample held at $\sim 10 \mathrm{~K}$. An external magnetic field $(0.44 \mathrm{~T}, 0.5 \mathrm{~T}$ or $0.90 \mathrm{~T}$ as indicated in the figure captions) was applied at $\sim 8^{\circ}$ off the surface, with the in-plane component aligned along the [100] direction of the MgO lattice. STM images were acquired in constant-current mode and all voltages refer to the sample voltage with respect to the tip.

Spin-polarized tip. The iridium STM tip was coated with silver by indentations into the Ag sample until the tip gave a good lateral resolution in the STM image. To prepare a spin-polarized tip, $\sim 1-5 \mathrm{Fe}$ atoms were each transferred from the $\mathrm{MgO}$ onto the tip by applying a bias voltage $(\sim 0.55 \mathrm{~V})$ while withdrawing the tip from near point contact with the Fe atom. The degree of spin polarization was verified by the asymmetry in $\mathrm{d} I / \mathrm{d} V$ spectra of Ti with respect to voltage polarity ${ }^{20}$.

RF measurement. The continuous wave electron spin resonance spectra were acquired by sweeping the frequency of an RF voltage $V_{\mathrm{RF}}$ generated by the RF generator (Agilent E8257D) across the tunneling junction and monitoring changes in the tunneling current. The current signal was modulated at $95 \mathrm{~Hz}$ by chopping $V_{\mathrm{RF}}$, which allowed the readout of the current by a lock-in technique ${ }^{17}$. The RF and $\mathrm{DC}$ voltages were combined at room temperature using an RF diplexer, and guided to the STM tip through semi-rigid coaxial cables with a loss of $\sim 30 \mathrm{~dB}$ at $20 \mathrm{GHz}^{17}$.

\section{Data availability}

The data that support the plots within this paper and other findings of this study are available from the corresponding authors upon reasonable request.

Received: 23 July 2020; Accepted: 15 January 2021; Published online: 12 February 2021 


\section{References}

1. Zhou, Y., Kanoda, K. \& Ng, T.-K. Quantum spin liquid states. Rev. Mod. Phys. 89, 025003 (2017).

2. Balents, L. Spin liquids in frustrated magnets. Nature 464, 199-208 (2010).

3. Christensen, N. B. et al. Quantum dynamics and entanglement of spins on a square lattice. Proc. Natl Acad. Sci. USA 104, 15264-15269 (2007).

4. Ma, X.-s. et al. Quantum simulation of the wavefunction to probe frustrated Heisenberg spin systems. Nat. Phys. 7, 399-405 (2011).

5. Nascimbène, S. et al. Experimental realization of plaquette resonating valencebond states with ultracold atoms in optical superlattices. Phys. Rev. Lett. 108, 205301 (2012).

6. Anderson, P. W. The resonating valence bond state in $\mathrm{La}_{2} \mathrm{CuO}_{4}$ and superconductivity. Science 235, 1196-1198 (1987).

7. Mourigal, M. et al. Fractional spinon excitations in the quantum Heisenberg antiferromagnetic chain. Nat. Phys. 9, 435-441 (2013).

8. Dalla Piazza, B. et al. Fractional excitations in the square-lattice quantum antiferromagnet. Nat. Phys. 11, 62-68 (2015).

9. Han, T.-H. et al. Fractionalized excitations in the spin-liquid state of a kagome-lattice antiferromagnet. Nature 492, 406-410 (2012)

10. Fu, M., Imai, T., Han, T.-H. \& Lee, Y. S. Evidence for a gapped spin-liquid ground state in a kagome Heisenberg antiferromagnet. Science 350, 655-658 (2015).

11. Hirobe, D. et al. One-dimensional spinon spin currents. Nat. Phys. 13, 30 (2016)

12. Vijayan, J. et al. Time-resolved observation of spin-charge deconfinement in fermionic Hubbard chains. Science 367, 186-189 (2020).

13. Hirjibehedin, C. F., Lutz, C. P. \& Heinrich, A. J. Spin coupling in engineered atomic structures. Science 312, 1021-1024 (2006).

14. Toskovic, R. et al. Atomic spin-chain realization of a model for quantum criticality. Nat. Phys. 12, 656-660 (2016).

15. Choi, D.-J. et al. Colloquium: atomic spin chains on surfaces. Rev. Mod. Phys. 91, 041001 (2019).

16. Khajetoorians, A. A., Wegner, D., Otte, A. F. \& Swart, I. Creating designer quantum states of matter atom-by-atom. Nat. Rev. Phys. 1, 703-715 (2019)

17. Baumann, S. et al. Electron paramagnetic resonance of individual atoms on a surface. Science 350, 417-420 (2015).

18. Choi, T. et al. Atomic-scale sensing of the magnetic dipolar field from single atoms. Nat. Nanotechnol. 12, 420-424 (2017).

19. Natterer, F. D. et al. Reading and writing single-atom magnets. Nature 543 , 226-228 (2017).

20. Yang, K. et al. Engineering the eigenstates of coupled spin-1/2 atoms on a surface. Phys. Rev. Lett. 119, 227206 (2017).

21. Bae, Y. et al. Enhanced quantum coherence in exchange coupled spins via singlet-triplet transitions. Sci. Adv. 4, eaau4159 (2018).

22. Yang, K. et al. Coherent spin manipulation of individual atoms on a surface. Science 366, 509-512 (2019).

23. Steinbrecher, M. et al. Quantifying the interplay between fine structure and geometry of an individual molecule on a surface. Preprint at https://arxiv.org/ abs/2007.01928 (2020).

24. Hirjibehedin, C. F. et al. Large magnetic anisotropy of a single atomic spin embedded in a surface molecular network. Science 317, 1199-1203 (2007).

25. Yang, $\mathrm{K}$. et al. Tuning the exchange bias on a single atom from $1 \mathrm{mT}$ to $10 \mathrm{~T}$. Phys. Rev. Lett. 122, 227203 (2019).

26. Slichter, C. P. Principles of Magnetic Resonance (Springer, 1996).

27. Loth, S. et al. Bistability in atomic-scale antiferromagnets. Science 335, 196-199 (2012).

28. Karbach, M., Hu, K. \& Müller, G. Introduction to the bethe ansatz II. Computers Phys. 12, 565-573 (1998).

29. Wootters, W. K. Entanglement of formation of an arbitrary state of two qubits. Phys. Rev. Lett. 80, 2245-2248 (1998).

30. Roscilde, T. et al. Studying quantum spin systems through entanglement estimators. Phys. Rev. Lett. 93, 167203 (2004).

31. Dehollain, J. P. et al. Nagaoka ferromagnetism observed in a quantum dot plaquette. Nature 579, 528-533 (2020).

32. Garlatti, E. et al. Portraying entanglement between molecular qubits with fourdimensional inelastic neutron scattering. Nat. Commun. 8, 14543 (2017).

33. Guidi, T. et al. Direct observation of finite size effects in chains of antiferromagnetically coupled spins. Nat. Commun. 6, 7061 (2015).

34. Gaita-Ariño, A., Luis, F., Hill, S. \& Coronado, E. Molecular spins for quantum computation. Nat. Chem. 11, 301-309 (2019).

35. Liu, J. et al. Electric field control of spins in molecular magnets. Phys. Rev. Lett. 122, 037202 (2019)

36. Luis, F. et al. Molecular prototypes for spin-based CNOT and SWAP quantum gates. Phys. Rev. Lett. 107, 117203 (2011)

37. Troiani, F. et al. Molecular engineering of antiferromagnetic rings for quantum computation. Phys. Rev. Lett. 94, 207208 (2005).
38. Santini, P., Carretta, S., Troiani, F. \& Amoretti, G. Molecular nanomagnets as quantum simulators. Phys. Rev. Lett. 107, 230502 (2011).

39. Hensgens, T. et al. Quantum simulation of a Fermi-Hubbard model using a semiconductor quantum dot array. Nature 548, 70-73 (2017).

40. Fujihala, M. et al. Gapless spin liquid in a square-kagome lattice antiferromagnet. Nat. Commun. 11, 3429 (2020).

41. Vojta, M., Mitchell, A. K. \& Zschocke, F. Kondo impurities in the Kitaev spin liquid: numerical renormalization group solution and gauge-flux-driven screening. Phys. Rev. Lett. 117, 037202 (2016)

42. Gomilšek, M. et al. Kondo screening in a charge-insulating spinon metal. Nat Phys. 15, 754-758 (2019).

43. Manousakis, E. The spin-1/2 Heisenberg antiferromagnet on a square lattice and its application to the cuprous oxides. Rev. Mod. Phys. 63, 1-62 (1991).

44. Zayed, M. E. et al. 4-spin plaquette singlet state in the Shastry-Sutherland compound $\mathrm{SrCu}_{2}\left(\mathrm{BO}_{3}\right)_{2}$. Nat. Phys. 13, 962-966 (2017).

45. Loth, S. et al. Measurement of fast electron spin relaxation times with atomic resolution. Science 329, 1628-1630 (2010).

46. Baltz, V. et al. Antiferromagnetic spintronics. Rev. Mod. Phys. 90, 015005 (2018).

47. Fukuhara, T. et al. Quantum dynamics of a mobile spin impurity. Nat. Phys. 9 , 235-241 (2013).

48. Gatteschi, D., Sessoli, R. \& Villain, J. Molecular Nanomagnets (Oxford Univ. Press, 2006)

\section{Acknowledgements}

We thank Bruce Melior for expert technical assistance; J.L. Lado and J. FernándezRossier for helpful discussions. We gratefully acknowledge financial support from the Office of Naval Research. S.-H.P., Y.B., T.E., P.W. and A.J.H. acknowledge support from Institute for Basic Science (IBS-R027-D1). A.A. acknowledges suppor from the Engineering and Physical Sciences Research Council (EP/L011972/1 and EP/P000479/1), the QuantERA European Project SUMO, and the European Union's Horizon 2020 research and innovation program under grant agreement No 863098 (SPRING).

\section{Author contributions}

K.Y. and C.P.L. designed the experiment. K.Y., S.-H.P., Y.B., T.E. and P.W. carried out the STM measurements. K.Y. and C.P.L. performed the analysis and wrote the manuscript with help from all authors. All authors discussed the results and edited the paper

\section{Competing interests}

The authors declare no competing interests.

\section{Additional information}

Supplementary information The online version contains supplementary material available at https://doi.org/10.1038/s41467-021-21274-5.

Correspondence and requests for materials should be addressed to K.Y., A.J.H. or C.P.L

Peer review information Nature Communications thanks Saw-Wai Hla and the other, anonymous, reviewer(s) for their contribution to the peer review of this work.

Reprints and permission information is available at http://www.nature.com/reprints

Publisher's note Springer Nature remains neutral with regard to jurisdictional claims in published maps and institutional affiliations.

\footnotetext{
(c) (i) Open Access This article is licensed under a Creative Commons Attribution 4.0 International License, which permits use, sharing adaptation, distribution and reproduction in any medium or format, as long as you give appropriate credit to the original author(s) and the source, provide a link to the Creative Commons license, and indicate if changes were made. The images or other third party material in this article are included in the article's Creative Commons license, unless indicated otherwise in a credit line to the material. If material is not included in the article's Creative Commons license and your intended use is not permitted by statutory regulation or exceeds the permitted use, you will need to obtain permission directly from the copyright holder. To view a copy of this license, visit http://creativecommons.org/ licenses/by/4.0/.
}

(c) The Author(s) 2021 